\title{
Note sur quelques espèces d'Amphibiens de l'Archipel Indo-Australien.
}

PA I

\section{Jean ROUX}

Conservateur du Muséum d'Histoire naturelle de Bâle.

En reprenant l'étude, à l'aide de divers travaux herpétologiques récents, de quelques Amphibiens de la collection du Muséum d'Histoire naturelle de Bâle, j'ai été conduit à modifier pour plusieurs espèces les déterminations faites antérieurement. Dans cette note, je désire consigner les résultats de mes observations sur quelques représentants de la famille des Ranidae.

\section{Rana lateralis Blgr.}

R. lateralis. Boubengen, G.-A. Ann. Mus. civ. Genova (2) Vol. 5, p. 483, Pl. 8, fig. 2. 1887.

id. Boulenger, G.-A. Fauna of British India. Rept., p. 457. 1890.

id. Laidlaw. Proc. zool. Soc. London, 1900, p. 886, Pl. 57, fig. 1 et 2 .

R. novae-britanniae (part.) Werner, Fr. Zool. Jahrb. (Syst.) Bd. 13, p. 493, Pl. 32, fig. 3. 1900.

La collection de Bâle renferme, parmi les espèces d'Amphibiens récoltées par G. Schneider, à Sumatra, et étudiées par Werner (loc. cit.), une Grenouille déterminée par ce dernier auteur comme $R$. novae-britanniae Wern. L'étude de ce spécimen m'a montré qu'il appartient en réalité à l'espèce que 
Boulenger a décrite sous le nom de $R$. lateralis. Cette espèce, connue tout d'abord de Birmanie (Tenasserim), a été retrouvée par Laidlaw (loc, cit.) dans le Siam méridional (Kelantan). L'exemplaire, récolté par Schneider, provient de Laut Tador, près de Deli, N. E. Sumatra. L'aire de dispersion de cette espèce se trouve notablement agrandie par cette trouvaille et intéresse donc l'Archipel Indo-australien à la faune duquel R. lateralis Blgr. doit être ajoutée. J'adjoindrai quelques notes à la description qu'a donnée Boulenger de cette forme.

Notre exemplaire est un mâle de $63^{\mathrm{mm}}$ de longueur (museauanus). Ce que Boulenger dit de la tête s'applique très bien à ce spécimen; le tympan est légèrement plus petit que l'œil. L'extrémité des doigts est faiblement dilatée; le bouton terminal, arrondi, n'est pas divisé par une rainure horizontale. Le premier doigt est plus long que le deuxième. Les orteils sont aux $3 / 4$ palmés, l'avant-dernière phalange du quatrième orteil est bordée d'un liséré membraneux et la dernière tout à fait libre. L'extrémité des orteils est élargie en petits disques qui sont partagés dans leur hauteur par une rainure horizontale. Les tubercules subarticulaires sont assez saillants; un fort tubercule métatarsien interne, en coussinet ovale allongé, mesure un peu moins de la demi-longueur de l'orteil. Ramené en avant le long du corps, le membre postérieur arrive, avec l'articulation tibio-tarsienne, entre l'oil et la narine.

La peau du dos n'est pas lisse, mais finement granuleuse ; outre les plis glandulaires latéraux, notre spécimen présente aussi les fines lignes glandulaires obliques sur le milieu du dos, que Laideaw a décrites. La coloration de cet individu est plus foncée que celle des exemplaires de la presqu'île malaise. La face supérieure est brune, les còtés du corps plus sombres que le dos; autour du tympan, une tache d'un brun noir; bord de la lèvre supérieure plus clair que le reste de la tète. Une glande humérale bien développée, marbrée de noir. Cette glande doit exister aussi chez l'exemplaire du Siam, si l'on en juge par le dessin de la planche accompagnant le travail de Laiduaw. Les membres ont des barres transversales d'un brun 
foncé, et la partie postérieure des cuisses est marbrée de noir et blanc. Face inférieure d'un gris sale, avec quelques dessins indistincts plus foncés sur la gorge. Le mâle possède deux sacs vocaux internes.

\section{Rana lireffti Blgr.}

R. lireffit. Boulevger. Calalog. Batrach. Sal. Ecaud., p. 64, Pl. 3, fig. 2. 1882.

id. Boulenger. Trans. zool. Soc. London, Vol. 12, p. 52.1886.

id. Boulenger. Ann. Mlag.nat. Hist. (9) Vol. 1, p. 241. 1918. R. nopae-britanniae. Werser. Zool. Anz., Bd. 17, p. 155. 1894. $i d$ Werser. Mitt. zool. Mus. Berlin, Bd. 1, p.111, Textfig. 1900.

Dans la collection de Bàle se trouve une Grenonille qui avait été déterminée comme Hyla nigrofrenata ${ }^{1}$ Gthr. et qui est en réalité un représentant du genre Rana, R. kreffti Blgr. Ellé provient de Nouvelle-Bretagne et répond parfaitement à la description qu'a donnée Werner de $R$. novae-britanniae. La comparaison de cette espèce avec $R$. kreffti Blgr. a montré, comme le supposait Boulenger, qu'il s'agit d'une seule et même espèce. Chez notre exemplaire, le tympan est plus petit que l'œil (environ ${ }^{2}{ }_{3}$ de la longueur de l'orbite). Les caractères tirés de la longueur du membre postérieur, de la membrane, des tubercules métatarsiens, coüncident parfaitement. Comme Werner l'a déja relevé la peau du dos est finement chagrinée.

\section{Rana modesta Blgr.}

R. modesta Boulexger. Catalogue Batr. Sal. Ecaud., p. 25, Pl. 1, fig. 3. 1882.

$i d$. Boulexger. Proc. zool. Soc. London, p. 228. 1897.

${ }_{1}$ Müller, Fr. 3. Nachtrag zum Katalog der herpetologischen Sammlung des Basler Museums, 1883 , p. 10. 
R. modesta Müller. Verhandl. naturf. Gesell. Basel, Bd. 10, p. 867.1895.

R. microtympanum. van Kanpen in : Weber, Zool. Ergebnisse einer Reise in Niederländ. Ost-Indien, Bd. 4, p. 386. 1907.

R. magna. Stejneger in : Smithson. Miscell. Collect., Vol. 52, p. 437. 1909.

Cette espèce, décrite tout d'abord du Nord de l'île de Célèbes, semble être plus répandue qu'on ne l'avait tout d'abord pensé. A còté d'une série de spécimens, provenant de cette région de l'île, la collection de Bâle possède un individu jeune, provenant de Bontorio (Sud-Célèbes). L'espèce que vas Kampen (loc. cit.) a nommée $R$. microtympanum et qui provient de Loka, près de Bonthain (Sud-Célèbes), nous paraît fondée sur de jeunes exemplaires de $R$. modesta. Cet auteur a, du reste, reconnu lui-même la parenté étroite qui existe entre ces deux formes, qui ne différeraient que par la grandeur du tympan et la longueur relative du premier et du deuxième doigt. Pour ce qui est de la dimension du tympan par rapport à celle de l'orbite, Boulenger a déjà indiqué, dans son second travail (1897), qu'elle pouvait varier dans une certaine mesure. J'ai pu m'en convaincre aussi $€ n$ examinant, soit des individus adultes, soit des jeunes de cette espèce. Chez quelques-uns d'entre eux, le tympan ne mesure pas plus du ${ }^{1 / 3}$ de la longueur de l'orbite. La distance séparant le tympan de l'œil est aussi sujette à varier. Quant au premier doigt, il n'est pas toujours plus long: que le deuxième, mais seulement aussi long que lui, ainsi que je l'ai observé chez des spécimens jeunes. Il me semble, d'après ce qui vient d'être dit, que l'espèce de van Kampen peut être incorporée à $R$. modesta. Cette dernière se trouve donc distribuée dans le Sud comme dans le Nord de Célèbes; mais sa distribution ne se borne pas à cette île, comme on l'avait cru tout d'abord. Vax Kampen (loc. cit., p. 385 et 386 ) indique comme localité l'île de Saleyer, au Sud de Célèbes (avec un certain doute, il est vrai, car il s'agit d'un jeune individu); cependant, 
cette trouvaille n'aurait rien d'étonnant si $R$. modesta se trouve répandue dans toute l'île de Célèbes. Le même auteur signale aussi cette espèce de Nusa Laut, petite île à l'Est d'Amboine, puis des îles Talaut, situées entre l'Archipel des Philippines et Célèbes. Etant donnée la présence de R. modesta à Talaut, on pouvait s'attendre à la trouver également aux Philippines. Or, Stejneger a décrit de Mindanao une Grenouille qu'il a appelée $R$. magna, voisine de $R$. macrodon. L'auteur américain ne possédant aucun spécimen de $R$. modesta, n'a pu comparer son espèce à cette dernière. Grâce à son obligeance, j'ai pu obtenir du Musée de Washington un bel exemplaire $\sigma^{x}$ adulte de R. magna et constaler sa parfaite identité avec l'espèce de Boulenger. Chez les exemplaires de Washington, la peau du dos est lisse; seule la région sacrale possède de petits tubercules pointus qu'on retrouve également sur les tibias. Chez quelques spécimens de notre collection, étudiés par Boulenger, ces tubercules sont aussi répartis sur la partie antérieure du dos, chez d'autres, ils sont moins développés. Chez les deux formes, l'extrémité des orteils est un peu élargie en boutons charnus qui ne semblent pas mériter le nom de "disques", car ils sont beaucoup moins aplatis que ceux de $R$. everetti, par exemple. La description de l'espèce de Stejneger s'applique très bien, sauf pour quelques variations individıelles, aux exemplaires de Célèbes. Stejneger signale cétte espèce des îles Mindanao, Basilan, Mindoro et Luzon, dans I'Archipel des Philippines.

\section{Rhacophorus leucomystax (Gravenh).}

R. leucomytax (Gravenh.). Boulenger, G. A. Proc. zool. Soc. London, p. 29. 1889.

Je rattache à cette espèce, très répandue dans le S.-E. du continent asiatique et dans la partie occidentale de l'Archipel Indo-Australien, un individu de notre collection qui portait le

${ }^{1}$ Monatsberichte Berlin. Akad., 1871, p. 580. 
nom de Polypedates raniceps Ptrs. ${ }^{1}$ Je dois dire d'emblée que je ne puis au juste indiquer si cette espèce est valide ou non, n'ayant pas vu les exemplaires types qui se trouvent au Musée de Berlin. Boulenger ne la mentionne pas dans son Catalogue. Fischer ${ }^{1}$ rapporte à l'espèce de Peters des spécimens provenant de Barabei, S.-E. de Bornéo. Un de ces exemplaires, donné par Fischer à Müller, pour la collection de Bâle, est mentionné par ce dernier auteur ${ }^{2}$ sous le nom de Rhacophorus Polyp.) raniceps Ptrs. Or, ce spécimen ne peut ètre rapporté à l'espèce décrite par Peters, mais il répond parfaitement à la description de Rhacophorus leucomystax Gravenh. J'ai comparé l'exemplaire en question, qui mesure $73^{\mathrm{mm}}$ du museau à l'anus, à l'espèce de Gravexhorst, dont la collection de Bâle possède de nombreux exemplaires et ai pu établir leur parfaite similitude. Seules, les taches foncées du dos sont peı accusées sur le spécimen étudié.

\section{Rhacophorus appendiculatus (Gthr.)}

R. appendiculatus (Günther). Boulenger, Cat. Batr. Sal., p. 86, Pl. 8, fig. 4. 1882.

R. phyllopygus. Werner. Zool.Jahrb. (Syst.) Bd. 13, p. 494, Pl. 32, fig. 5. 1900.

Notre collection possède le type de l'espèce décrite de Sumatra par Werner, espèce qui ne semble pas pouvoir être séparée de $R$. appendiculatus (Gthr.). Les petites différences notées entre les deux formes, ne sont pas suffisantes pour les séparer spécifiquement; le canthus rostralis est un peu plus anguleux et l'espace interorbitaire un peu plus large que chez $R$. appendiculatus $\left(1^{1 / 3}\right.$ au lieu de $1^{1 / 2}$ comme l'indique Werner). Les tubercules sous-articulaires ne sont doubles que sous le quatrième orteil, mais Boulenger a déjà fait cette observation

${ }^{1}$ Arch. f. Naturgeschichte, Vol. 51, p. 43. 1885.

2 Verhandl, naturf. Gesell. Basel, Bd. 8, p. 256. 1887. 
sur des exemplaires des îles Mentawei, ainsi que l'a relevé SCHENKEL ${ }^{1}$.

\section{Rhacophorus pardalis Gthr.}

R. pardalis. Boulenger. Catal. Batr. Sal., p. 91.1882.

R. pulchellus Gthr. Werner. Zool. Jahrb. (Syst.) Bd. 13, p. 495, Pl. 33, fig. 7. 1900.

L'espèce de Sumatra, décrite par Werner sous le nom de R. pulchellus et dont le type se trouve à Bâle, doit ètre réunie à l'espèce de Günther. L'individu de Sumatra est un $\sigma^{x}$ qui me paraît encore jeune. Le tympan est un peu plus petit que ne l'indique Boulenger, il mesure la moitié de la longueur de l'orbite, et les disques des doigts sont un peu plus petits que le tympan. L'espace interorbitaire est un peu plus large que la paupière supérieure. J'ai constaté la même coloration sur les côtés du corps chez les deux formes; les appendices cu- . tanés de l'avant-bras, du tarse, du talon et le long de l'orteil sont, de même, développés d'une manière toute semblable.

1 Verhandl. naturf. Gesell., Basel, Bd. 13, p. 149. 1901. 


\section{$2 \mathrm{BHL}$ Biodiversity Heritage Library}

Roux, J. 1918. "Note sur quelques espèces d'amphibiens de l'Archipel Indo-Australien." Revue suisse de zoologie 26, 409-415.

https://doi.org/10.5962/bhl.part.16169.

View This Item Online: https://www.biodiversitylibrary.org/item/40641

DOI: https://doi.org/10.5962/bhl.part.16169

Permalink: https://www.biodiversitylibrary.org/partpdf/16169

\section{Holding Institution}

MBLWHOI Library

Sponsored by

MBLWHOI Library

\section{Copyright \& Reuse}

Copyright Status: NOT_IN_COPYRIGHT

This document was created from content at the Biodiversity Heritage Library, the world's largest open access digital library for biodiversity literature and archives. Visit BHL at https://www.biodiversitylibrary.org. 